\title{
Progress and Gratitude
}

I am pleased to report that the submission rate for new manuscripts has risen linearly from 121 papers in 2000 to 290 in 2008. Innovations, such as the Reflections section, Critically Appraised Topics and historical reviews, have been well received, as have the changes to the cover. We have added 32 members to the Editorial Review Board and hope this will enhance the quality of papers, based on their reviews and contributions.

I am delighted that our initiative to enhance the Electronic Continuing Professional Development (e-CPD) component of the Journal was approved at the Board level of the Canadian Neurological Sciences Federation (CNSF) meeting in June in Halifax. This will allow us to move forward with this important component of the web-based Journal. It will provide our members with enhanced educational opportunities. The initial phases under development include: Grand Rounds; Clinical Neuropathological Conferences, videos of movement disorders, epilepsy cases, neurosurgical operations, Diagnostic Challenges, Ask-the-Expert opportunities, and lots more. We shall have a demo presentation for you in the near future, likely in 2010 .

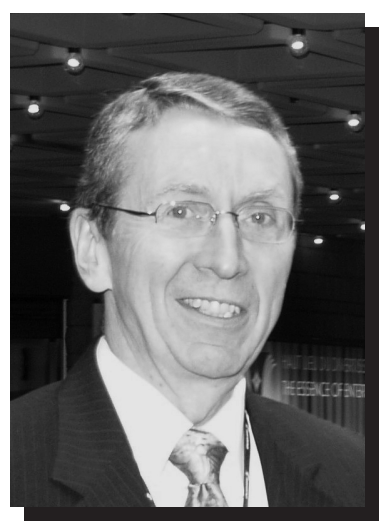

Another important proposal approved by the CNSF is the plan to allow open access to the Journal by select medical schools and teaching hospitals in China, Cuba and India. This will provide needed knowledge transfer to these developing countries and will encourage their talented neuroscientists to submit their basic and clinical research papers to the Journal.

Dr. Vivek Mehta, an Edmonton neurosurgeon, was presented the Distinguished Reviewer of the Year Award, at the June CNSF Congress in Halifax. We congratulate and thank him for his work for the Journal.

I want to thank those who have worked hard for these progressive steps, including Lisa Bicek, Jorge Burneo, David Chan, Jim Cummings, Max Findlay, Robert Hammond, Mary Jenkins, Mandar Jog, Cindy Leschyshyn, Maggie McCallion, Dan Morin, Michael Poulter, Brian Wheelock, Doug Zochodne and other members of the Editorial Board and Editorial Review Board as well as the CNSF for its support, even in tough economic times. It is good to move forward with support from such good and dedicated people.
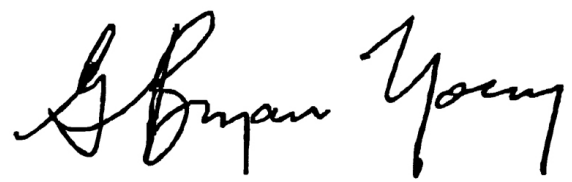

G. Bryan Young, Editor-in-Chief 